\title{
Behandlung der proximalen Patellafixation des Pferdes mit Akupunktur
}

\section{Eine risilkoarme und erfolgreiche Therapieform}

\author{
Isabell Herold, Thomas Kreis
}

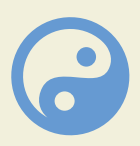

\section{Zusammenfassung}

Es wurde untersucht, wie erfolgreich die Behandlung der proximalen Patellafixation mit Akupunktur ist. Dazu wurde an 18 Patienten mit habitueller oder permanenter Patellafixation das Yin- und YangFersengefäß mit Akupunktur des Ni 6 und Bl 62 geöffnet. Bei 15 der 18 Patienten trat eine sofortige Besserung ein, 2 Patienten waren erst nach einer Woche asymptomatisch. Ein Patient zeigte keinen Behandlungserfolg. Somit erscheint die Akupunktur als erfolgreiche und risikoarme Methode bei proximaler Patellafixation gut geeignet.

\section{Einleitung}

In der Pferdepraxis sieht man sich häufig mit der habituellen Patellafixation des Pferdes konfrontiert. Die Invasivität der konventionellen Behandlungsmethoden scheint dabei nicht in Relation zu ihrem Erfolg zu stehen. Deshalb wurde untersucht, wie erfolgreich eine standardisierte Behandlung mit Akupunktur ist.

\section{Anatomie}

Die Patella bildet mit dem medialen und lateralen Rollkamm des Femurs das Kniescheibengelenk (Art. femoropatellaris). Simultan mit der Bewegung des Kniekehlgelenkes (Art. femorotibialis) erfolgt in diesem Schlittengelenk eine Gleitbewegung der Patella zwischen den beiden Trochleae des Femur.

Für den Mechanismus der Patellafixation sind die drei Kniescheibenbänder von entscheidender Bedeutung. Dabei handelt es sich um das mediale, mittlere und laterale Kniescheibenband (Ligg. patellae medialis, intermedius und lateralis).

Die Kniescheibe ist als Sesambein in den M. quadriceps femoris einbezogen.
Dabei bildet die Endsehne seines mittleren Muskelbauches (M. rectus femoris) das mittlere Kniescheibenband. Der Vastus lateralis des M. quadriceps femoris endet im lateralen Kniescheibenband und der Vastus medialis entsprechend im medialen Kniescheibenband. Diese drei Kniescheibenbänder sind etwa auf gleicher Höhe an der Patella verankert. Dabei findet das mediale Kniescheibenband seinen Ursprung am Fibrocartilago parapatellaris medialis, dem knorpeligen Fortsatz an der medialen Seite der Patella.

Diese Bänder verlaufen konvergierend zur Tibia. Das mediale Kniescheibenband endet auf der Tuberositas tibiae, das mittlere im Sulcus tuberositas tibiae und das laterale inseriert am Margo cranialis der Tibia [5] (ه Abb. 1).

Als laterale Oberschenkelmuskulatur nehmen der M. tensor fasciae latae, der Vastus lateralis des M. quadriceps femoris

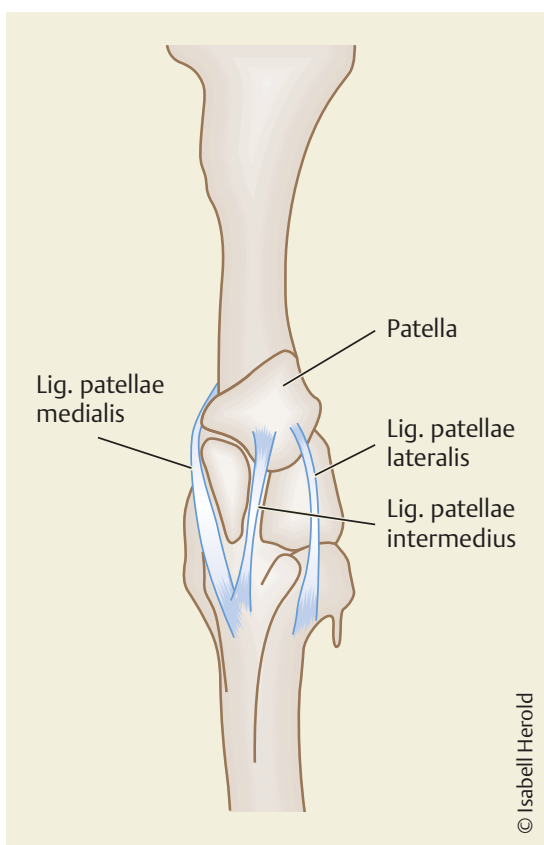

Abb. 1 Patellabänder beim Pferd. und der M. biceps femoris Einfluss auf das Femoropatellargelenk und inserieren alle in das laterale Kniescheibenband. Diese Muskelgruppe übt also einen Zug nach proximolateral auf die Kniescheibe aus. Als Gegenspieler nehmen auf der medialen Seite der M. sartorius, der M. gracilis, der Vastus medialis des M. quadriceps femoris und der M. semimembranosus Verbindung mit dem medialen Kniescheibenband auf und ziehen die Patella nach proximomedial [5].

\section{Physiologie des Patellarmechanismus}

Das mittlere Kniescheibenband, der Fibrocartilago parapatellaris medialis und das mediale Kniescheibenband bilden eine Schlaufe, die sich am medialen Rollkamm des Femurs einhaken kann. Dadurch ist die Patella fixiert und das Kniegelenk in Streckstellung arretiert. Durch die Spannsägenkonstruktion ist nun nicht nur das Knie-, sondern auch das Sprunggelenk versteift, und das Pferd kann so nahezu ohne Aufwand von aktiver Muskelkraft stehen.

Zur Fixation der Patella wird sie durch Kontraktion des M. rectus femoris nach proximal gezogen und durch Zug der medialen Oberschenkelmuskulatur (s.o.) leicht nach medial rotiert und hakt sich so auf den Trochanter medialis. Daher wird dieser Mechanismus auch als proximale Patellafixation bezeichnet.

Der Patellarmechanismus wird aufgelöst durch die Kontraktion des M. rectus femoris. Dieser zieht die Patella wieder nach proximal, und der Zug der lateralen Oberschenkelmuskulatur (s.o.) hakt die Patella nach lateral wieder aus.

Für das „Einhaken“ ist also die mediale und für das „Aushaken“ die laterale Oberschenkelmuskulatur verantwortlich [6,7]. 


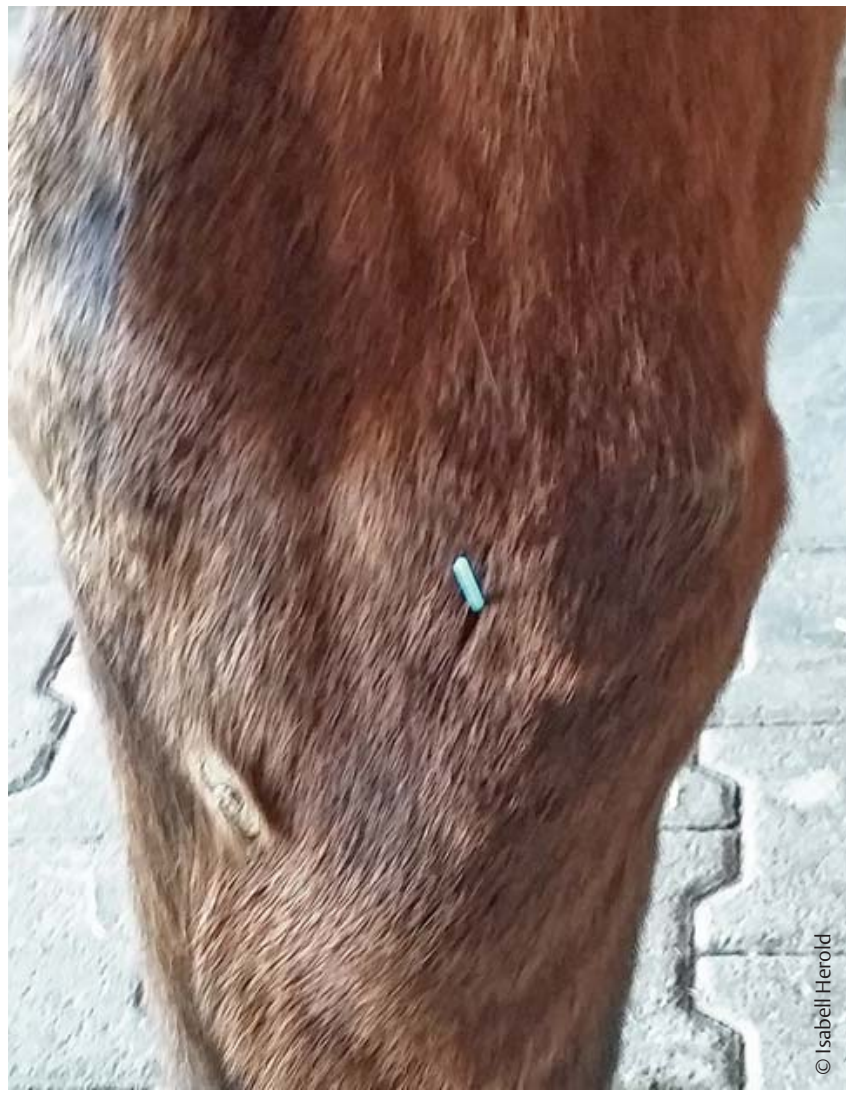

Abb. 2 Akupunktur des Ni 6.

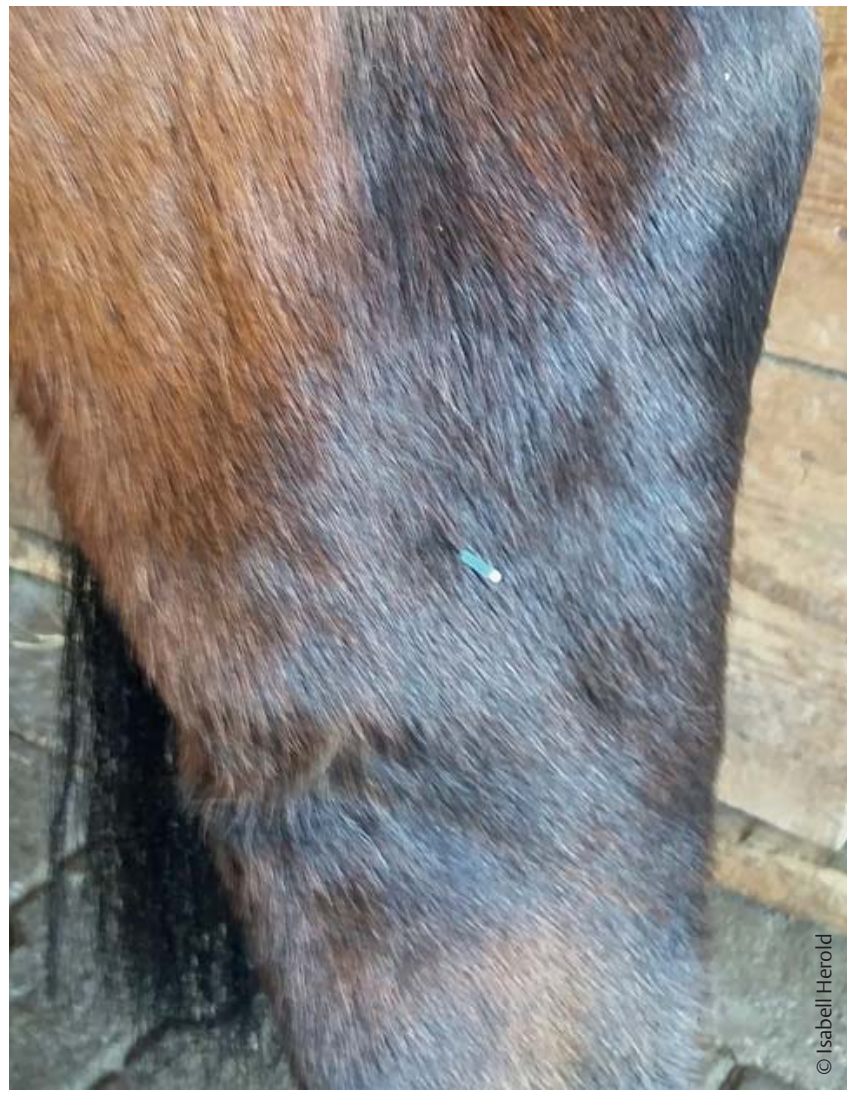

Abb. 3 Akupunktur des Bl 62.

\section{Pathologie des Patellarmechanismus}

Im Zustand der akuten Patellafixation bleibt die Kniescheibe auf dem medialen Rollkamm des Oberschenkels fixiert und Knie- und Sprunggelenk können nicht gebeugt werden. Das in Streckung fixierte Bein wird im Fesselgelenk oft in Flexionsstellung gehalten, sodass die Hufspitze über den Boden streift. Dieser Zustand kann über mehrere Tage, wenige Stunden (permanente Patellafixation) oder in der Bewegung nur sehr kurz (habituelle Patellafixation) auftreten. Er löst sich oft von selbst, um in unterschiedlichen Abständen von wenigen Schritten oder mehreren Tagen wieder aufzutreten. Bei der chronischen Form tritt eine habituelle oder permanente Patellafixation wiederholt auf.

Die Therapie bei einer akuten permanenten Patellafixation besteht im Rückwärts- oder Seitwärtsrichten des Pferdes, wobei die Patella mit der Hand nach lateroproximal oder mediodistal gedrückt wird. Die zwangsweise Bewegung eines Pferdes mit akuter Patellafixation kann zu einer Abrissfraktur der Tuberositas tibiae führen und sollte vermieden werden [7].
Diese proximale Patellafixation ist $\mathrm{zu}$ differenzieren von Patellaluxationen nach lateral oder seltener nach medial oder distal, die jedoch zu völlig anderen Symptomen führen.

Die konventionelle Therapie der chronisch-rezidivierenden Patellafixation besteht in der Desmotomie des medialen Kniescheibenbandes, um ein Einhaken zu verhindern. Ebenfalls werden Injektionen von Irritantia an das mediale Kniescheibenband durchgeführt, um es in der nachfolgenden Entzündung zu verkürzen. Beide Methoden sind nicht nur invasiv und schmerzhaft, sondern auch mit einer hohen Rezidivrate behaftet. Zudem treten mehrere Monate nach der Desmotomie des medialen Kniescheibenbandes häufig Knorpelläsionen und Frakturen an der distalen Patella auf [8]. Deshalb erscheinen dem Autor die konventionellen Therapien unbefriedigend. Um die Therapie durch Akupunktur verständlicher zu machen, soll kurz auf die biomechanische Ätiologie eingegangen werden.

Pferde mit sehr geradem Hinterbein sind prädisponiert für die habituelle Patellafixation. Aber auch bei Pferden ohne
Prädisposition tritt diese Erkrankung auf, wenn die Muskulatur entweder gerade stark abgebaut oder stark aufgebaut wird. In voll auftrainiertem oder voll abtrainiertem Zustand der Oberschenkelmuskulatur bessert sich die Symptomatik meist. Neuere Studien lassen darauf schließen, dass die habituelle Patellafixation durch eine Imbalance der Kräfteverhältnisse der inneren und äußeren Oberschenkelmuskulatur entsteht. Schuurman et.al. sprechen sogar von einer Überaktivität des Vastus medialis des M. quadriceps femoralis als Ursache der Patellafixation [6]. Da die innere Oberschenkelmuskulatur für das „Einhaken“ verantwortlich ist, ist diese relativ zu stark und die äußere Oberschenkelmuskulatur zum „Aushaken“ relativ zu schwach.

So wäre es denkbar, dass sich in der Phase des Abtrainierens die äußere Muskulatur mit ihrer größeren Muskelmasse verhältnismäßig schneller abbaut als die innere. Ist diese Phase abgeschlossen, stehen die Kräfteverhältnisse wieder in richtiger Relation und die Symptomatik bessert sich. 
Wird das Pferd auftrainiert, tritt der umgekehrte Effekt ein: bis die kräftige äußere Oberschenkelmuskulatur ihr volles Maß erreicht hat, sind die vergleichsweise kleineren Muskeln der inneren Oberschenkelmuskulatur schon ausgebildet.

Diese Imbalance der inneren und äußeren Oberschenkelmuskulatur eröffnet die Möglichkeit, diese Erkrankung mit Akupunktur über das Yin- und Yang-Schreitgefäß (Yinqiaomai und Yangqiaomai) zu behandeln.

\section{Das Yin- und Yang-Schreitgefäß (Yinqiaomai und Yangqiaomai)}

Diese beiden Sondermeridiane kontrollieren den Muskeltonus der Beine und stehen in regem Austausch und enger Verbindung zueinander. Sie werden in der Humanmedizin eingesetzt, „um die Spannung der Beinmuskeln der inneren und äußeren Seite auszugleichen“ [4].

Das Yin-Schreitgefäß oder auch YinFersengefäß entspringt an der Innenseite der Ferse, steigt an der Innenseite des Oberschenkels zu den Genitalien und verläuft durch Abdomen und Thorax des Pferdes bis zum inneren Augenwinkel. Dieser Meridian wird mit Niere 6 (Ni 6) geöffnet. Ni 6 befindet sich distal des Malleolus medialis der Tibia. Dieser Punkt ist nicht nur der Öffner des Yin-Schreitgefäßes, sondern ist auch Mittel der Wahl, um das Nieren-Yin zu stärken $[2,4]$ (๑ Abb. 2).

Das Yang-Schreitgefäß (Yang-Fersengefäß) zieht als Ausläufer des Blasenmeridians von der Außenseite der Ferse über den äußeren Oberschenkel und seitlich am Rumpf bis zum inneren Augenwinkel und von dort bis Gallenblase 20. Der Öffnungspunkt dieses Meridians ist Blase 62 (Bl 62). Bl 62 befindet sich distal des Malleolus lateralis der Tibia und damit direkt gegenüber von Ni 6 (\$Abb. 3). Seine vorrangige Wirkung bezieht sich auf die Öffnung des Yang-Schreitgefäßes.

\section{Material und Methoden}

Über einen Zeitraum von 14 Monaten wurden 18 Pferde mit einer habituellen Patellafixation mit Akupunktur der Öffnungspunkte des Yin- und Yang-Schreitgefäßes behandelt ( $\$$ Abb. 2 und 3).

Die Pferde waren zwischen 2 und 23 Jahren alt, es handelte sich um 11 Stuten und 8 Wallache. Die Patienten gliederten sich auf in 1 Shetlandpony, 2 Isländer und 15 Warmblüter. Als Voraussetzung in diese Gruppe aufgenommen zu werden, mussten die Pferde lahmfrei sein und nach Angaben der Besitzer mehrmals wöchentlich eine habituelle oder permanente $\mathrm{Pa}$ tellafixation zeigen.

Nach abgeschlossener Lahmheitsuntersuchung erfolgte der Untersuchungsgang zur Akupunktur. 17 der 18 Patienten zeigten hierbei einen palpationsdolenten $\mathrm{Ba}$ Shen. Dieser Punkt war uni- oder bilateral schmerzhaft. Ba Shen entspricht dem Punkt Blase 54 und liegt in einer Muskeldelle in der Mitte einer Linie von Bai Hui (Spatium lumbosacrale) und dem Trochanter major femoris. Dieser diagnostische Punkt deutet auf Schmerzen und Verspannungen der Hinterhand. Andere Ergebnisse des Untersuchungsganges stimmten nicht überein und zeigten auch keinen signifikanten Zusammenhang.

Die Pferde wurden beidseits zunächst am Bl 62 und anschließend beidseits am Ni 6 genadelt. Es wurden Akupunkturnadeln der Größe $0,2 \times 15 \mathrm{~mm}$ senkrecht eingestochen. Am Ende der einsetzenden
Entspannung der Pferde oder bei beginnender Unruhe, wurden die Nadeln in umgekehrter Reihenfolge wieder gezogen. Die Verweildauer der Nadeln betrug zwischen 5 und 10 Minuten.

\section{Ergebnisse}

Nach 14 Tagen wurden die Patienten wieder vorgestellt. Nach Angaben der Besitzer war die Patellafixation bei 15 Pferden schon am Tag nach der Behandlung nicht mehr aufgetreten, zwei Pferde zeigten innerhalb der ersten Woche zunächst nur eine Besserung der Symptomatik und waren erst in der zweiten Woche nach der Behandlung asymptomatisch. Bei allen Pferden war der Ba Shen nun ohne Schmerzhaftigkeit. Diese Pferde wurden nur ein einziges Mal behandelt. Ein Isländerwallach zeigte eine von der Behandlung nicht beeinflusste Symptomatik und litt auch nach mehrmaliger, identischer Behandlung noch unter mehrfach wöchentlich auftretenden habituellen Patellafixationen. 
Bei 13 Pferden trat innerhalb von 10 Monaten kein Rezidiv auf. 3 Pferde zeigten 6 bis 12 Monate später eine erneut auftretende habituelle Patellafixation und wurden noch einmal auf gleiche Weise erfolgreich behandelt. Ein Pferd wurde verkauft und konnte deshalb nach 10 Monaten nicht nachuntersucht werden.

\section{Diskussion}

Eine Imbalance des Muskeltonus der äußeren zur inneren Oberschenkelmuskulatur als Ursache der Patellafixation wurde schon in 2009 in Bad Wildungen von Thomas Kreis geäußert. Seine Arbeitsgruppe behandelt diese Erkrankung erfolgreich mit einer Injektion eines Lokalanästhetikums in den Ba Shen.

Diese Hypothese der Imbalance wird von den anatomischen Grundlagen und der Biomechanik der Patella sowie Studien wie die von Schuurman et.al. [6] gestützt.

Die Behandlung der habituellen Patellafixation über das Yin- und Yang-Schreitgefäß bietet sich also theoretisch an, hat praktisch guten Erfolg und könnte in einigen Fällen für Patient und Tierarzt risikoärmer sein als die Injektion in den Ba Shen.

$\mathrm{Zu}$ Beginn der Versuchsphase dieser Methode wurde eine 15-jährige Patientin sowohl mit Bl 62 und Ni 6 beidseits als auch mit einer Nadelung des Ba Shen auf der schlechteren Seite behandelt. Es trat eine deutliche Verschlechterung der Symptome auf, die schließlich mit einer erneuten Behandlung nach 14 Tagen von Bl 62 und Ni 6 ohne Ba Shen wieder verschwand. Eine eindeutige Entscheidung zwischen den beiden Methoden scheint also ratsam.

Alle Patienten zeigten im Untersuchungsgang zur Akupunktur die unterschiedlichsten Befunde. Nur aus Rücksicht auf möglichst repräsentative Ergebnisse dieser Versuchsreihe wurde darauf verzichtet, diese Befunde vorher oder simultan mit zu behandeln. Das Yin- und YangSchreitgefäß haben keinen diagnostischen Punkt und werden daher symptombezo- gen angewendet [4]. Auf die Nadelung der Kopplungspunkte Lu 7 und Dü 3 wurde verzichtet.

Ein erhöhter Muskeltonus der Innenseite ist Symptom einer Fülle des Yin qiao mai. Als Therapie wird das Öffnen des Yang qiao mai empfohlen [2]. Es wäre Inhalt einer weiteren Untersuchung, ob das alleinige Stechen von Bl 62 schon ausreichend wäre, diesen guten Erfolg bei der Therapie der habituellen Patellafixation zu erreichen.

\section{Summary \\ Treatment of Equine Upward Patellar Fixation by Acupuncture}

This study examined the success of the treatment of upward patellar fixation by acupuncture. 18 patients, suffering from habitual or permanent patella fixation were treated with acupuncture of renal 6 and bladder 62, opening the Yin qiao mai and Yang qiao mai. 15 of the 18 patients were asymptomatic the next day, 2 patients improved one week after treatment. One horse did not show response to acupuncture at all. Therefore, acupuncture seems to be a successful and low-risk method in the treatment of upward patella fixation.

Key words

upward patella fixation - horse - acupuncture Ni 6 - Bl 62

\section{Literatur}

[1] Ashdown RR, Done S. Topographische Anatomie des Pferdes. Stuttgart: Enke Verlag; 1988

[2] Gösmeier I, Friese L. Handout Praxisseminar Akupunktur Pferd Teil V: Außerordentliche Meridiane. Oer-Erkenschwick; 2015

[3] Guray JY, v.d. Bosch E. Praxis der Pferdeakupunktur. Stuttgart: Sonntag Verlag; 2002

[4] Maciocia G. Grundlagen der chinesischen Medizin. 2. Aufl. München: Urban \& Fischer; 2008

[5] Nickel R, Schummer A, Seiferle E. Lehrbuch der Anatomie der Haustiere, Band I Bewegungsapparat. 5. Aufl. Berlin: Paul Parey Verlag; 1984

[6] Schuurman S, Kersten W, Weijs WA. The equine hind limb is actively stabilized during standing. Journal of Anatomy 2003; 202: 355362
[7] Stashak TS. Adam's Lahmheit bei Pferden. 4. Aufl. Hannover: M.\&H. Schaper; 1989

[8] Wissdorf H, Gerhards H, Huskamp B, Deegen E. Praxisorientierte Anatomie und Propädeutik des Pferdes. 3. Aufl. Hannover: M.\&H. Schaper; 2010

Online zu finden unter

http://dx.doi.org/10.1055/s-0042-110731

() Dr. med. vet. Isabell Herold

Praktische Tierärztin

Bürg 27

83627 Warngau

E-Mail: isa@herold-simon.de

Studium in Budapest und Berlin (1990-1996); Assistenz in verschiedenen Pferdepraxen und -kliniken (1996-2000); seit 2000 in Gemeinschaftspraxis für Pferde am Tegernsee mit Ehemann Dr. Tilman Simon; 2 Söhne. Co-Autorin Praxisleitfaden Zahn- und Kiefererkrankungen des Pferdes (Simon, Herold, Schlemper, Paul Parey, 2008); mehrere Veröffentlichungen zum Thema Pferdezahnheilkunde; Zusatzbezeichnung und Tätigkeitsschwerpunkt Akupunktur bei chronischen Erkrankungen des Pferdes.

Dr. Thomas Kreis

Industriestr. 41

53359 Rheinbach

E-Mail: thkreisvet@aim.com

Jahrgang 1958 in Ludwigshafen, 1977-1980 Studium (D), 1977-1980 Studium Agrar und Jazzgitarre an der Austin Peay State University Tennessee; 1980-1985 Studium der Veterinärmedizin an der JLU Giessen; seit 1986 Tierarzt in eigener Praxis. Seit 1998 Weiterbildung auf den Gebieten Akupunktur und Traditionelle Chinesische Medizin u. a. bei O. Kothbauer, A. Zohmann, U. Petermann, J. Y. Guray, E. van den Bosch, A. Rösti; 2001 Ausbildung Manuelle Medizin beim Pferd bei Detlef Rittmann; 2002 Ausbildung in Pferde-Osteopathie bei Dominique Giniaux; seit 2003 Dozent am Vierbeiner Reha Zentrum/Private Akademie für erweiterte Tiermedizin in Bad Wildungen mit den Schwerpunkten Akupunktur, Traditionelle Chinesische Medizin, Manuelle Medizin beim Pferd. Neben der klassischen Schulmedizin intensiver Einsatz von Physiotherapie, Neuraltherapie und der Goldimplantation bei Kleintier und Pferd. 2004 Zuerkennung der Zusatzbezeichnung Akupunktur; 2011 Leiter des Vierbeiner Reha Zentrums Bad Wildungen gemeinsam mit A. Zohmann; Vorträge und Seminare zu den Themenkreisen Akupunktur sowie Manuelle Medizin/Osteopathie. 